\title{
Trajectory studies for SPEAR3 LTB
}

\author{
Yipeng Sun, James Safranek and Kai Tian \\ SLAC National Accelerator Laboratory, Menlo Park, California 94025, USA
}

April 11, 2013

\begin{abstract}
In this note, trajectory stability in SPEAR3 Linac-to-booster (LTB) transport line is studied. It is observed that an electron gun body temperature variation or a linac RF phase variation may introduce beam energy variation which then translates into a trajectory change after certain dipole magnets. Long time BPM resolution is also investigated, plus a calculation on the initial beam angle variation. Beam trajectory is controlled for a good injection efficiency into the booster, by using the measured response matrix of certain steering correctors. Dispersion is also measured for a future implementation of a beam energy feedback.
\end{abstract}

\section{Trajectory stability}

LTB is the transport beamline from the SPEAR3 linac to its booster ring. It consists of three dipole magnets, six quadrupole magnets between the dipoles, one sextupole corrector magnet [1] after B2 (the second dipole). The beam diagonostic system includes six beam position monitors (BPM) and several beam screens. There are four vertical steering correctors, three horizontal steering correctors, plus three dipole-trim horizontal correctors which are attached on the main dipoles. A sketch of the LTB beamline is shown in Figure 1.

The trajectory stability in the LTB beamline is dependent on two factors. The first is the initial beam condition which includes the beam position, angle and energy jitter. The second factor is the boundary condition of the LTB beamline, such as the variation of the magnetic fields in and near the LTB beamline (for example in booster), the variation of the steering corrector strength, and so on.

During the past several accelerator physics studies in February-March 2013, long term trajectory stability in LTB is investigated. The repetition rate is always $10 \mathrm{hz}$. After excluding the possibility of B1 (the first dipole) current variation, linac RF power and phase variation, it is concluded that the periodic horizontal trajectory oscillation after B1 might be correlated with the electron gun body temperature [2], as shown in Figure 2.

Another example is shown in Figure 3, where the transverse trajectory jump in and after LTB is possibly introduced by a linac RF phase jump.

\footnotetext{
*Email: yisun@slac.stanford.edu
} 
The BPM resolution is also calculated using beam trajectory information in 600 shots, as shown in Figure 4. The BPM resolution is below $350 \mu \mathrm{m}$ and $200 \mu \mathrm{m}$ for horizontal and vertical plane, respectively.

As discussed above, the horizontal trajectory variation after B1 is from beam energy variation. This is also proved by a comparison between horizontal and vertical trajectory (the dipoles bend in horizontal plane), as shown in Figure 5. The vertical trajectory jump in BPM5 and BPM6 indicates that there might be a vertical dispersion leak out of LTB. A shot-by-shot analysis of correlation between BPM1-2 and BPM1-3 indicates that the initial beam angle variation is small, as shown in Figure 6 .

\section{Trajectory feedback}

The goal of LTB trajectory feedback is to ensure a good injection efficiency into the downstream booster ring. Here a straightforward way is employed, which uses $\mathrm{C} 4 \mathrm{~V}$ (C5H) and C5V (B3T) to control the vertical (horizontal) trajectory in BPM5 and BPM6. The beam position and angle into booster septum is then fixed. The target BPM5 and BPM6 readings is set to be an empirical value which has been observed with good injection efficiency into booster.

The required steering correctors' strength change is related with the target beam position change, via the response matrix, as shown in Formulae (1) and (2) for linear case, also in Formulae (3) for a coupled case.

$$
\begin{gathered}
\left(\begin{array}{ll}
R_{12(1)} & R_{12(2)} \\
R_{12(3)} & R_{12(4)}
\end{array}\right) \cdot\left(\begin{array}{c}
x_{1}^{\prime} \\
x_{2}^{\prime}
\end{array}\right)=\left(\begin{array}{c}
\Delta x_{5} \\
\Delta x_{6}
\end{array}\right) \\
\left(\begin{array}{ll}
R_{34(1)} & R_{34(2)} \\
R_{34(3)} & R_{34(4)}
\end{array}\right) \cdot\left(\begin{array}{c}
y_{1}^{\prime} \\
y_{2}^{\prime}
\end{array}\right)=\left(\begin{array}{c}
\Delta y_{5} \\
\Delta y_{6}
\end{array}\right) \\
\left(\begin{array}{llll}
R_{12(1)} & R_{12(2)} & R_{14(1)} & R_{14(2)} \\
R_{12(3)} & R_{12(4)} & R_{14(3)} & R_{14(4)} \\
R_{32(1)} & R_{32(2)} & R_{34(1)} & R_{34(2)} \\
R_{32(3)} & R_{32(4)} & R_{34(3)} & R_{34(4)}
\end{array}\right) \cdot\left(\begin{array}{c}
x_{1}^{\prime} \\
x_{2}^{\prime} \\
y_{1}^{\prime} \\
y_{2}^{\prime}
\end{array}\right)=\left(\begin{array}{c}
\Delta x_{5} \\
\Delta x_{6} \\
\Delta y_{5} \\
\Delta y_{6}
\end{array}\right)
\end{gathered}
$$

These response matrix were measured experimentally by scanning the associated steering corrector strength in steps, as shown in Figure 7 and Figure 8. For each step fifty beam shots were averaged to minimize the impact from BPM noise.

Using the above discussed response matrix, a trajectory feedback code is prepared in MATLAB [3]. It controls the beam position and angle at the septum to ensure a good injection efficiency into the booster. On April 9th, 2013 this code was successfully tested in the accelerator physics studies, as shown in Table 1 and Figure 9 below. The target BPM readings for an empirically good injection efficiency are listed as 'step 1' in Table 1. The trajectory near the septum was then manually changed by 2-4 mm, which was done by changing the strength of $\mathrm{C} 1 \mathrm{~V}$ and $\mathrm{C} 2 \mathrm{H}$ (upstream steering correctors). After that there is no injection into the booster, and the Q-meter reads zero as shown in Figure 9. Then the trajectory feedback code was launched, which corrected the trajectory in BPM5 and BPM6, also recovered injection into booster, as shown in Figure 9. 
Table 1: BPM5 and BPM6 readings: original setting for a good injection efficiency (step 1); after manually tweak $\mathrm{C} 1 \mathrm{~V}$ and $\mathrm{C} 2 \mathrm{H}$ to lose injection (step 2); after running trajectory feedback code (step 3).

\begin{tabular}{l|c|c|c}
\hline & step 1 & step 2 & step 3 \\
\hline BPM5(X) [mm] & 2.6918 & 3.9728 & 2.789 \\
BPM5(Y) [mm] & 0.1433 & 3.7979 & 0.18845 \\
BPM6(X) [mm] & 2.4442 & 4.0405 & 2.5478 \\
BPM6(Y) [mm] & 0.6136 & 4.3888 & 0.58069 \\
\hline
\end{tabular}

\section{Energy feedback}

Besides the low level RF control system, beam-based energy feedback is also proposed. The horizontal trajectory response is employed to correct possible energy jitter from upstream linac, by tuning back the linac K3 power. This study is still on going.

For the aim of beam-based energy feedback, horizontal dispersion was measured at BPM1-6 by changing linac K3 RF power. The measured dispersion with and without B2 sextupole correction is shown in Figure 10 below. The maximum dispersion is found to be at BPM2 which could be used as energy feedback. Second order dispersion leak is observed at BPM5 and BPM6.

In case that B2 sextupole corrector being turned off, the dispersion change in BPM4 indicates a non-zero beam trajectory in B2 sextupole component (otherwise should only change second order dispersion).

\section{Acknowledgement}

The author would like to thank X. Huang, S. Gierman, J. Schmerge and J. Sebek for helpful discussions.

This work was supported by the DOE under Contract DE-AC02-76SF00515.

\section{References}

[1] Kai Tian, SPEAR AP note 32, http://ssrl.slac.stanford.edu/ets/slaconly/documentcontrol/ssrlap/apnotes.htm (2011).

[2] John Schmerge and Stephen Gierman, private communication (2013).

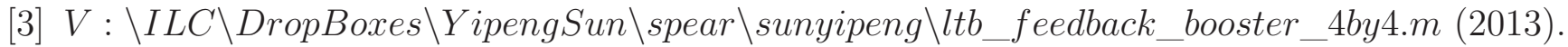




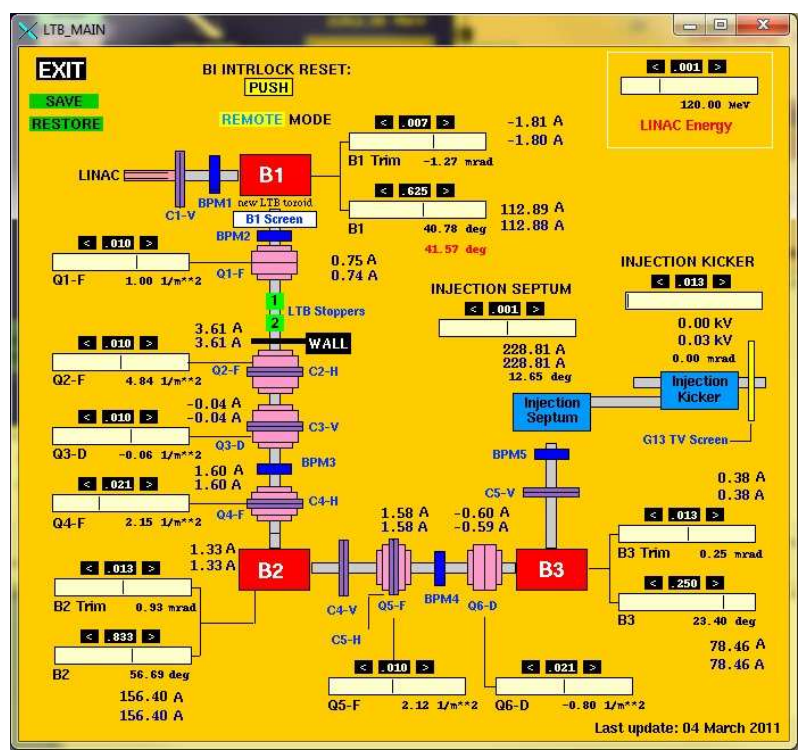

Figure 1: Sketch of the LTB beamline.
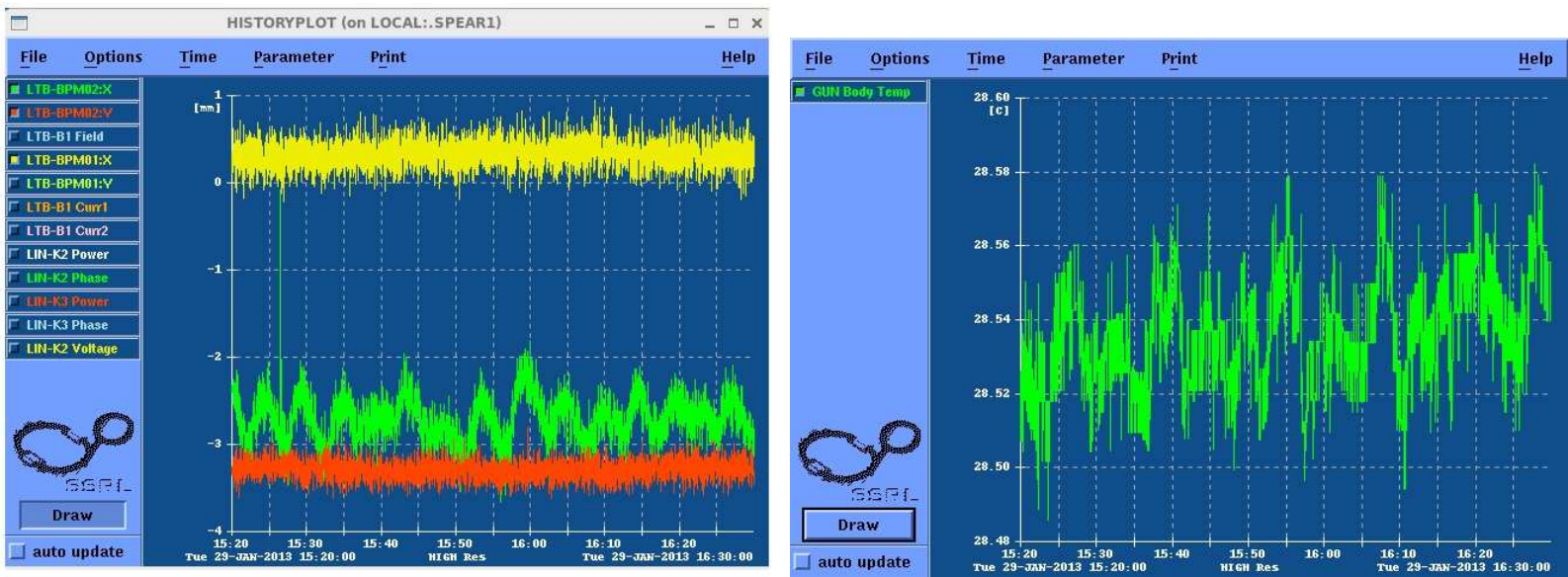

Figure 2: History plot of BPM2 horizontal trajectory (left) and electron gun body temperature (right) shows possible correlation on beam energy change between these two. 

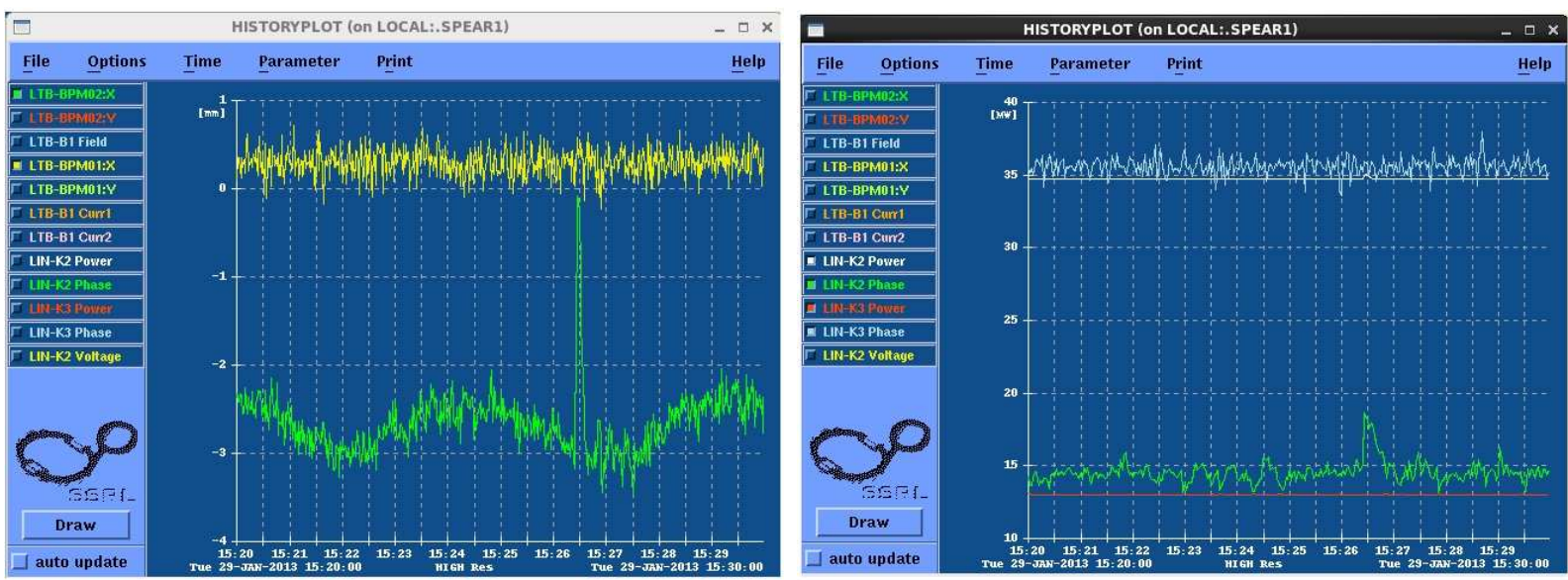

Figure 3: History plot of BPM2 horizontal trajectory (left) and linac K2 phase (right) shows change of beam energy and downstream $\mathrm{x}$ trajectory at the same time.
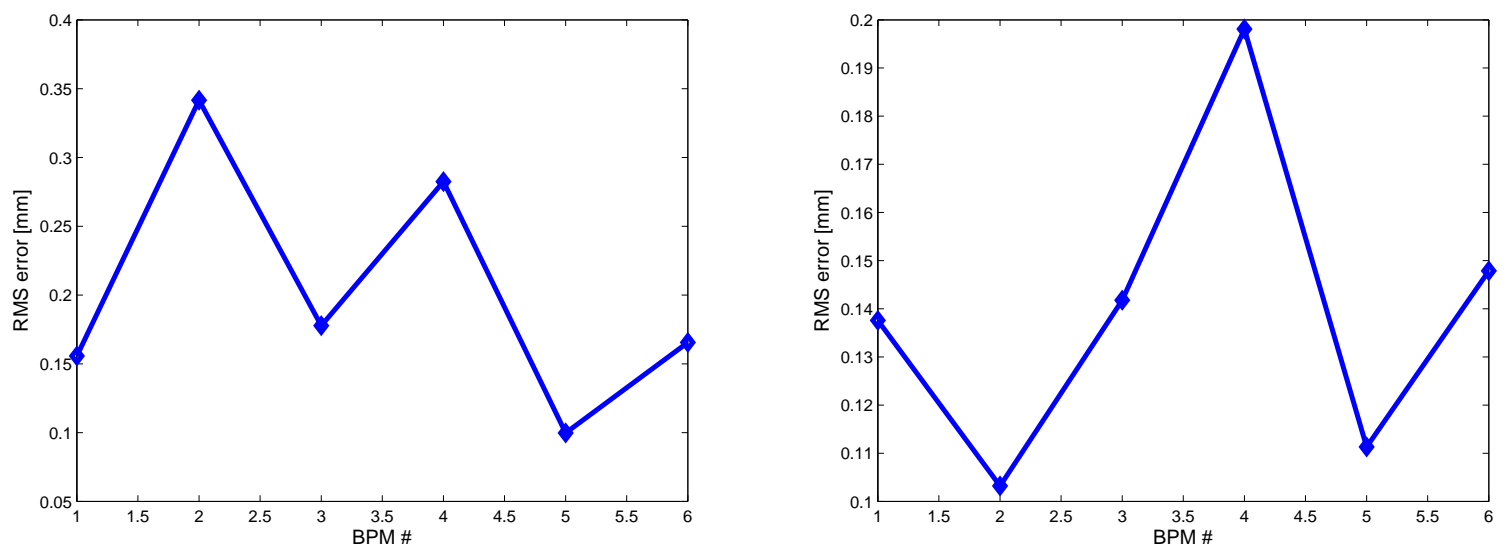

Figure 4: Statistical analysis of BPM1-6 X (left) and Y (right) readings for 10 minutes (600 shots), showing the RMS deviation of each BPM. 

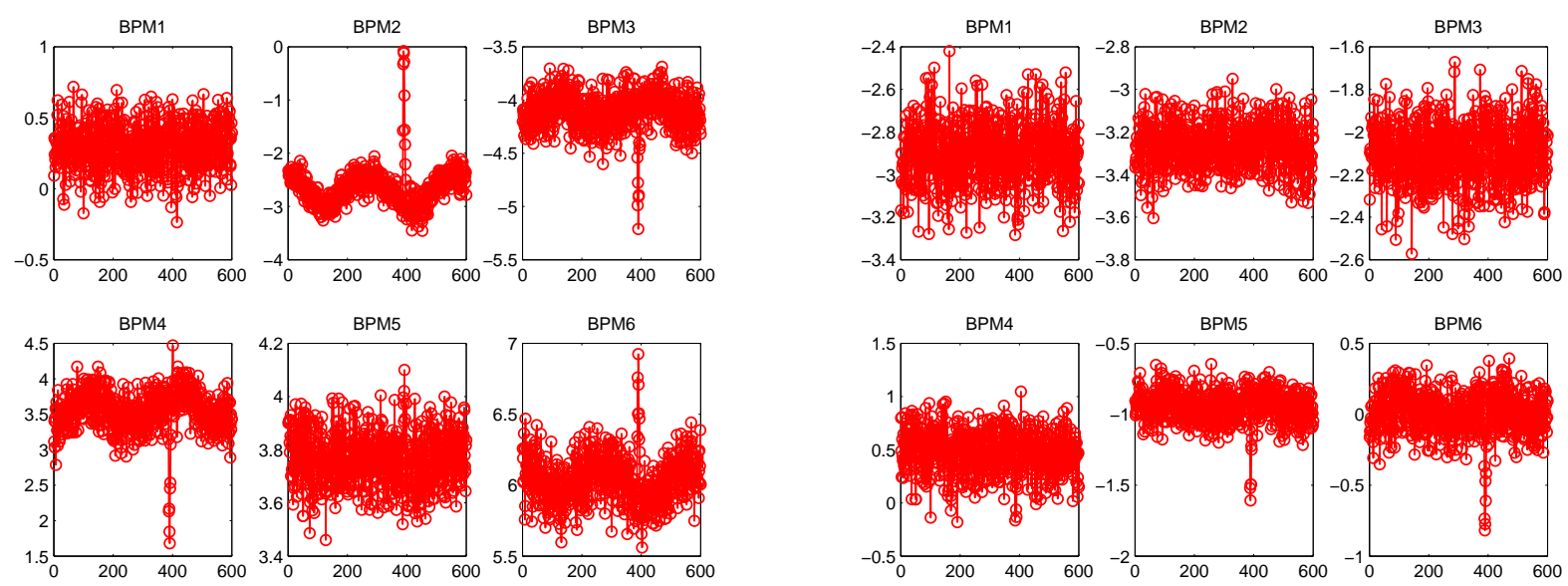

Figure 5: Horizontal (left) and vertical (right) trajectory of BPM1-6 in 10 minutes.
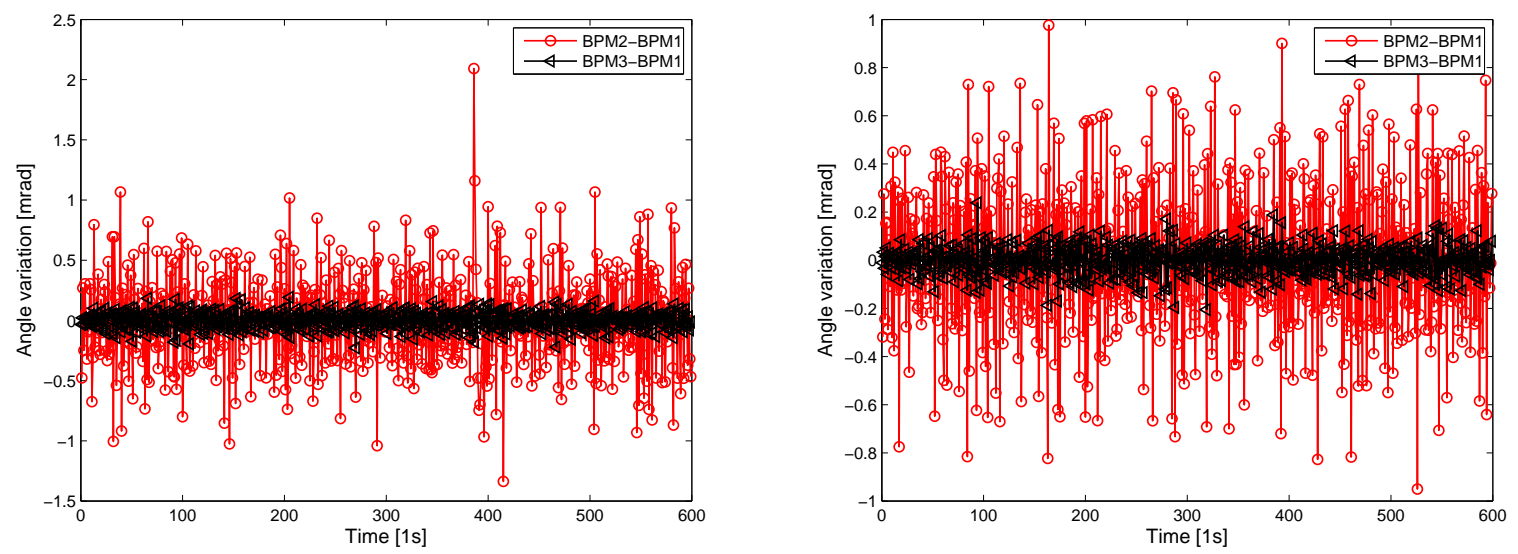

Figure 6: Initial beam angle variation from upstream linac, as calculated by a correlation between BPM1-2, also BPM1-3, for horizontal (left) and vertical (right) trajectory. 

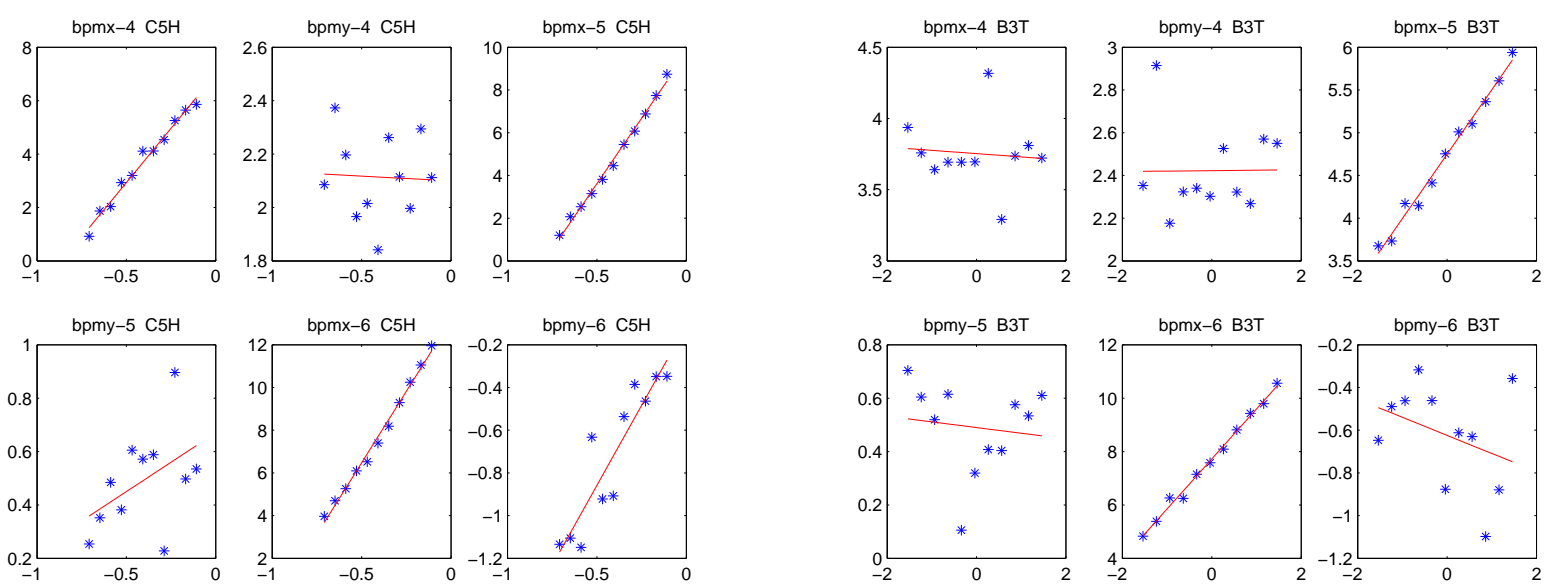

Figure 7: Response matrix measurement of C5H (left) and B3T (right).
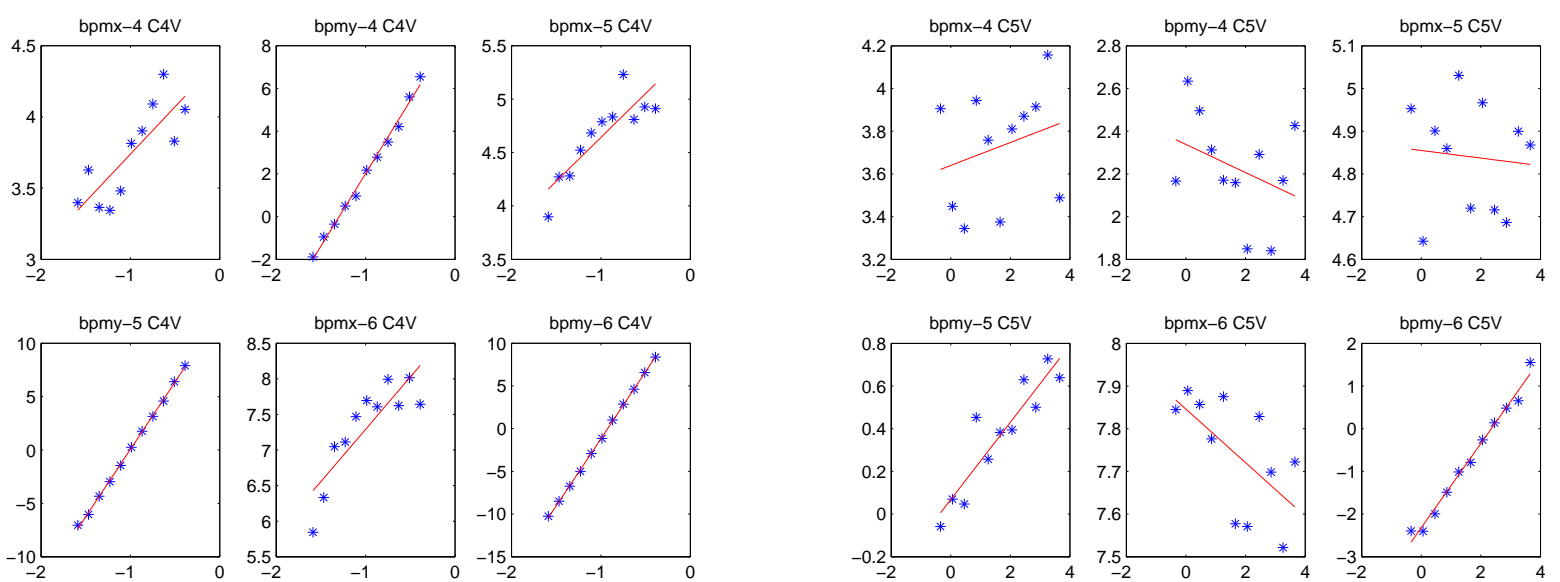

Figure 8: Response matrix measurement of C4V (left) and C5V (right). 


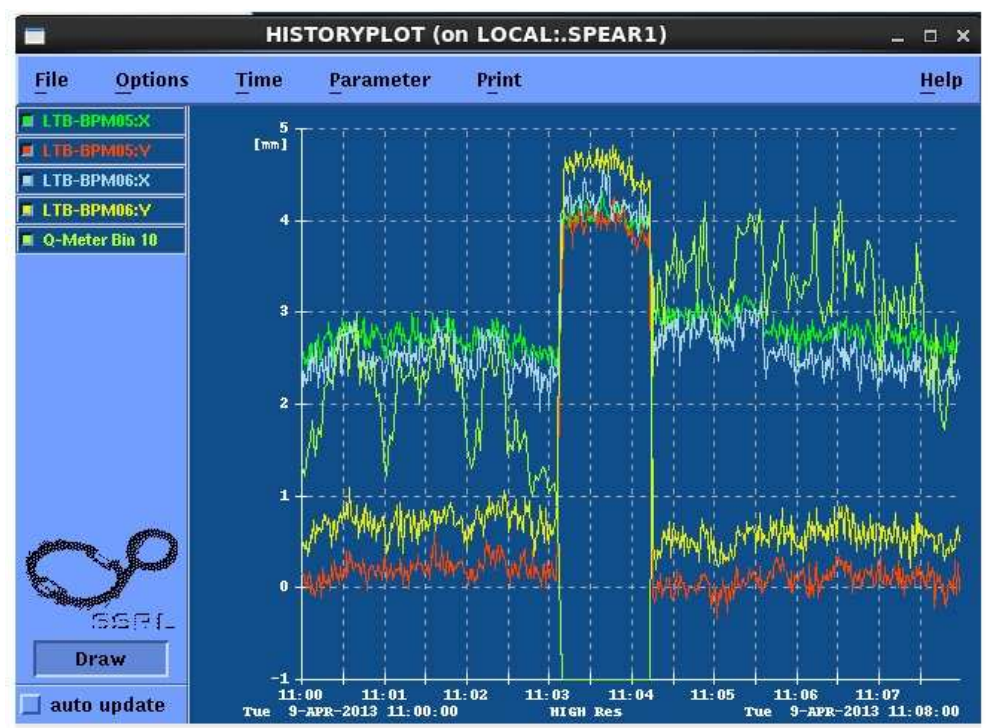

Figure 9: History plot of BPM5 and BPM6 readings, plus Q-meter data in booster (accumulated beam current), show that the trajectory feedback code works as expected.
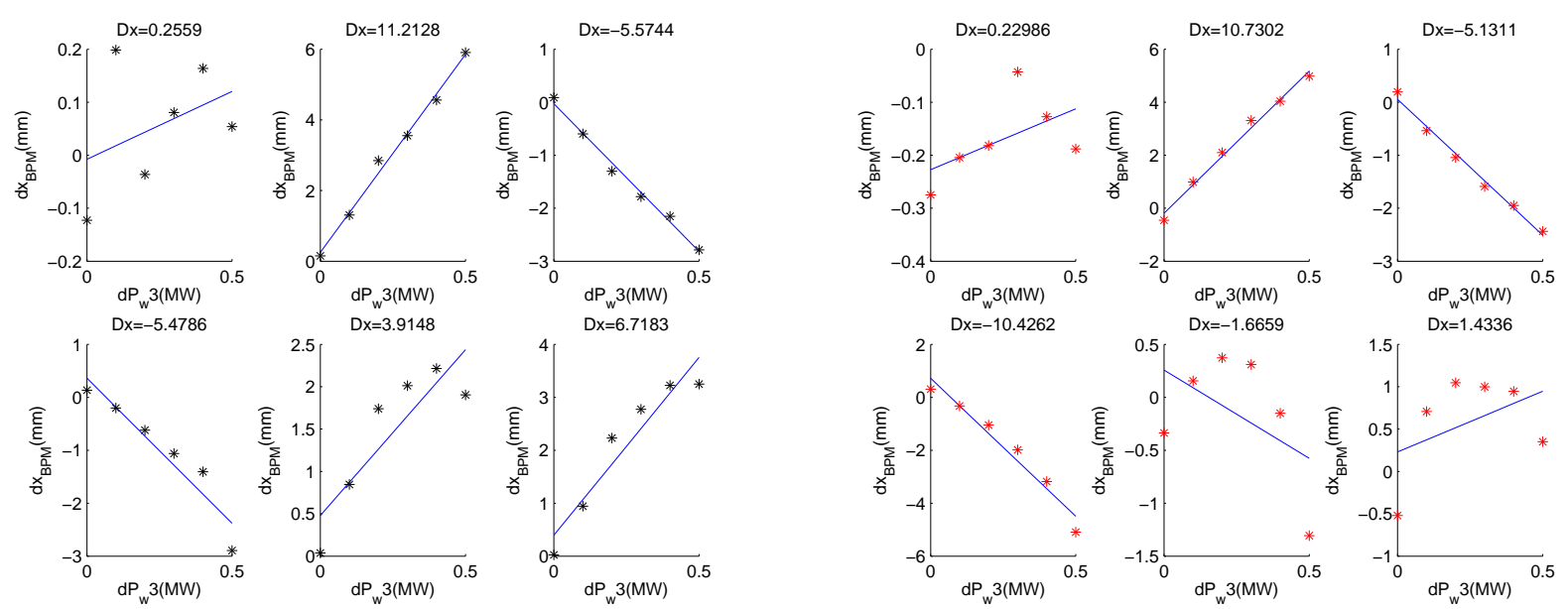

Figure 10: Dispersion measurement at BPM1-6, with (left) and without (right) B2 sextupole corrector. 